\title{
Respiratory Consequences of Pediatric Post-COVID Syndrome: Case Series
}

\author{
Karolina Dolezalova ${ }^{1}$, Jana Tukova ${ }^{2}$, and Petr Pohunek ${ }^{3}$ \\ ${ }^{1}$ Fakultní Thomayerova nemocnice \\ ${ }^{2} \mathrm{VFN}$ \\ ${ }^{3}$ Charles University, 2nd School of Medicine
}

October 28, 2021

\begin{abstract}
We report data on pediatric post-COVID syndrome from a Czech multicentric observational study. We enrolled children aged 218 years with persisting respiratory symptoms (cough, dyspnoea, chest pain) more than twelve weeks after COVID-19. Medical history, physical examination, lung function testing, chest X-ray and blood tests were performed. In 39 enrolled children, dominant reported symptoms were exercise-induced dyspnoea and cough. On lung function testing, we found abnormal results in less than $15 \%$ of children and on imaging in less than $20 \%$. From the analysis of enrolled subjects, we identified four possible post-COVID subgroups: "standard", "asthmatic", "sporty" and "polymorphous". Complete remission of tracked symptoms occurred within a median of 4 months in followed subjects
\end{abstract}

\section{Introduction}

Long-term sequelae following coronavirus 2019 infection (COVID-19) have been extensively described in adult population. Diagnosis of post-COVID syndrome is mainly reserved for clinical features persisting more than twelve weeks after an acute COVID-19 and not explained by an alternative diagnosis. About 10\% of adults in an ambulatory setting and about $70 \%$ of previously hospitalized people reported miscellaneous general or more specified symptoms three months after COVID-19 onset. These included fatigue, fever, pain, or neurological, respiratory, cardiovascular, gastrointestinal, dermatologic, musculoskeletal, and other organ symptoms. Although meta-analyses confirmed lower incidence and severity of acute pediatric COVID19 , great attention is devoted to severe complications following infection in a pediatric population known as multisystem inflammatory syndrome in children (MIS-C), so-called pediatric inflammatory multisystem syndrome temporally associated with SARS-CoV-2 (PIMS-TS). Syndrome develops within four weeks after COVID 19 exposure and is associated with a high risk of multiorgan failure, especially with severe cardiac involvement [6]. Pediatric post-COVID syndrome represents different diagnosis focused on long-lasting symptoms. Only limited data regarding incidence, clinical picture, diagnosis, therapy, or prognosis of pediatric post-COVID are available. Recently, several case series recruited from random parental contact of physician by internet web social media forum, survey from patients of pediatric departments,cohorts of randomly selected school classes with population-based seronegative control group single center cohort examined by phone call and outpatient assessment and prospective cohort study based on self-data reporting by a mobile application were published.

The objective of our study was to explore clinical picture, severity, and prognosis of post-COVID syndrome in children with a dominant focus on the respiratory system. Children were referred and followed up by the national network of Czech pediatric pulmonologists. Additionally, we suggested and tested a panel of screening tests for diagnosis and evaluation of structural and functional anomalies of the respiratory tract in pediatric post-COVID. 


\section{Methods}

This prospective national observational study targeted centers for pediatric pulmonology from all regions of the Czech Republic. From January to June 2021, 11 pediatric pulmonologists enrolled all children aged 2 to 18 years with persistent respiratory symptoms after COVID-19 referred by pediatricians from inpatient or outpatient settings. Inclusion criteria were: (1) presence of dyspnea at rest or on exertion, cough, or chest pain twelve or more weeks after infection with severe acute respiratory syndrome coronavirus 2 (SARS-CoV-2); and (2) documentation of preceding SARS-CoV-2 infection by positive PCR test or detection of significant elevation of antibodies; and (3) absence of any pre-existing chronic respiratory disease. We applied a standardized protocol to evaluate structural and functional anomalies and exclude alternative diagnoses. Initially, medical history, physical examination, lung function testing comprising spirometry, fraction of exhaled nitric oxide (FeNO), diffusing capacity (DLCO), and 6-minute walk test (6MWT), plain chest radiograph, and blood tests were performed in all enrolled children. Laboratory tests comprised blood count, basic biochemistry and allergy panel, D-dimer levels, and SARS-CoV-2 antibodies. In children with abnormal results of the initial panel, we considered individually an extended spectrum of investigative methods: chest ultrasound, spiral chest CT scan with high-resolution reconstruction, CT-pulmonary angiograms, ventilation/perfusion (VP) lung scan. For all enrolled subjects, we scheduled at least two outpatient visits within six months.

We compared numerical variables using One-way ANOVA and categorical variables with Fisher's exact test. All analyses were performed using IBM SPSS Statistics software version 21. This study was approved by the Ethics Committee with multicentric competence (ID A-21-01).

\section{Results}

In total, 40 children fulfilled the inclusion criteria between January and June 2021. One boy was excluded due to positive paired serology for Mycoplasma pneumoniae. In 39 children (median age $13.5 \pm 4.8$ years, IQR: 8-15 years, boys $43.6 \%)$, COVID-19 was confirmed by positive PCR test in $35(89.7 \%)$ and by serology in $14(35.9 \%)$. Exertional dyspnea $(76.9 \%)$ followed by chronic cough $(48.7 \%)$ represented dominant symptoms, while dyspnea at rest and chest pain were less prevalent (30.8\% resp. 17.9\%) (see Table 1). Half of the children $(53.8 \%)$ reported more than one symptom. Single abnormal results of lung function tests, chest X-ray, or D-dimers were seen in 15 children $(38.5 \%)$, in 3 children $(7.7 \%)$ we found a combination of two pathological results. There was no association between any symptoms and abnormal results. In 9 subjects, we suspected incipient bronchial asthma coinciding with COVID-19 infection based on personal and family histories, spirometry, laboratory results, and therapeutic effect of anti-asthma treatment. After data collection, we recognized four strikingly different subgroups of post-COVID manifestation in children: "standard", "asthmatic", "sporty" and "polymorphous". In subgroup of 5 "sporty" children with high-intensity training, no dyspnea at rest was noticed, and we saw physiological results of all diagnostic tests except for 1 abnormal chest X-ray. In other five children who suffered from various "polymorphous" symptoms, we observed a trend to higher prevalence of dyspnea at rest and/or fatigue compared to other participants (Fisher's exact test $\mathrm{p}=0.056)$.

In 34/39 subjects followed for the whole duration of our study (1 lost from follow-up, 4 with current follow-up $<6$ months), complete remission of tracked symptoms occurred within a median of 4 months (range 1.5-8, see Table 2.). We observed two unusual courses of the disease in our group. A 15-year-old boy with pleural pain, pleural effusion confirmed by lung ultrasound, normal ventilation/perfusion lung scan and normal Ddimers, and complete remission within seven months after first post-COVID symptoms. An 18-year-old girl with hemoptysis during acute COVID had abnormal 6MWT, physiologic chest CT scan, and D-dimers and complete remission within six months (Table 3).

Table 1 Symptoms and abnormal results in all subjects and post-COVID subgroups 


\begin{tabular}{|c|c|c|c|c|c|c|c|c|c|}
\hline & $\begin{array}{l}\text { Gender } \\
\text { (male) }\end{array}$ & Symptoms & Symptoms & Symptoms & Symptoms & $\begin{array}{l}\text { Abnormal } \\
\text { func- } \\
\text { tional or } \\
\text { labora- } \\
\text { tory } \\
\text { results }\end{array}$ & $\begin{array}{l}\text { Abnormal } \\
\text { func- } \\
\text { tional or } \\
\text { labora- } \\
\text { tory } \\
\text { results }\end{array}$ & $\begin{array}{l}\text { Abnormal } \\
\text { func- } \\
\text { tional or } \\
\text { labora- } \\
\text { tory } \\
\text { results }\end{array}$ & $\begin{array}{l}\text { Abnormal } \\
\text { func- } \\
\text { tional or } \\
\text { labora- } \\
\text { tory } \\
\text { results }\end{array}$ \\
\hline \multirow[t]{2}{*}{$\begin{array}{l}\text { All } \\
(\mathrm{n}=39)\end{array}$} & \multirow[t]{2}{*}{$17(43.6)$} & Cough & $\begin{array}{l}\text { Dyspnea } \\
\text { at rest }\end{array}$ & $\begin{array}{l}\text { Exertional } \\
\text { dyspnea }\end{array}$ & $\begin{array}{l}\text { Chest } \\
\text { pain }\end{array}$ & Spirometry & $6 \mathrm{MWT}$ & TLCO & $\begin{array}{l}\text { Chest } \\
\text { X-ray }\end{array}$ \\
\hline & & $\begin{array}{l}19 \\
(48.7 \%)\end{array}$ & $\begin{array}{l}12 \\
(30.8 \%)\end{array}$ & $\begin{array}{l}30 \\
(76.9 \%)\end{array}$ & $\begin{array}{l}7 \\
(17.9 \%)\end{array}$ & $\begin{array}{l}5 \\
(13.2 \%)\end{array}$ & $\begin{array}{l}3 \\
(8.8 \%)\end{array}$ & $\begin{array}{l}3(11.5 \\
\%)\end{array}$ & $\begin{array}{l}7 \\
(18.9 \%)\end{array}$ \\
\hline $\begin{array}{l}\text { Standard } \\
\text { post- } \\
\text { COVID } \\
(\mathrm{n}=20)\end{array}$ & $11(55)$ & $9(45.0 \%)$ & $6(30.0 \%)$ & $\begin{array}{l}16 \\
(80.0 \%)\end{array}$ & $5(25.0 \%)$ & $3(15 \%)$ & $2(11.1 \%)$ & $1(8.3 \%)$ & $4(22.2 \%)$ \\
\hline $\begin{array}{l}\text { Asthmatic } \\
\text { subgroup } \\
(\mathrm{n}=9)\end{array}$ & $5(55.5)$ & $6(66.7 \%)$ & $3(33.3 \%)$ & $6(66.7 \%)$ & 0 & $2(25 \%)$ & 0 & $2(40 \%)$ & $2(22.2 \%)$ \\
\hline $\begin{array}{l}\text { Sporty } \\
\text { subgroup } \\
(\mathrm{n}=5)\end{array}$ & $1(20)$ & $2(40.0 \%)$ & 0 & $4(80.0 \%)$ & $1(20.0 \%)$ & 0 & 0 & 0 & $1(20 \%)$ \\
\hline \multicolumn{2}{|c|}{$\begin{array}{l}\text { Polymorpho@is }(0) \\
\text { subgroup } \\
(\mathrm{n}=5)\end{array}$} & $2(40.0 \%)$ & $3(60.0 \%)$ & $4(80.0 \%)$ & $1(20.0 \%)$ & 0 & $1(25 \%)$ & 0 & 0 \\
\hline \multicolumn{2}{|l|}{$\begin{array}{l}\text { Fisher's } \\
\text { exact } \\
\text { test }{ }^{[?]}\end{array}$} & $\mathrm{p}=0.7$ & $\mathrm{p}=0.25$ & $\mathrm{p}=0.9$ & $\mathrm{p}=0.3$ & $\mathrm{p}=0.7$ & $\mathrm{p}=0.45$ & $\mathrm{p}=0.26$ & $\mathrm{p}=0.8$ \\
\hline
\end{tabular}

Data presented as numbers (proportions, \%).

DLCO - diffusing capacity of the lungs for carbon monoxide, 6MWT - 6-minute walk test

${ }^{[?]}$ Differences of categorical variables such as symptoms and abnormal results between subgroups were compared by Fisher's test

Table 2 Persistence of reported symptoms in months within post-COVID until remission

\begin{tabular}{lll}
\hline Subgroup & Duration in months & One-way ANOVA \\
\hline Standard post-COVID 16/20 & $5.0( \pm 1.5)$ & $\mathrm{p}=0.7$ \\
Asthmatic subgroup 8/9 & $4.1( \pm 1.9)$ & \\
Sport subgroup 4/5 & $4.9( \pm 1.8)$ & \\
Polymorphous subgroup 5/5 & $4.5( \pm 2.0)$ & \\
\hline
\end{tabular}

Data expressed as mean $( \pm \mathrm{SD}),{ }^{[?]}$ evaluation of subjects with complete follow-up

Table 3 Details and clinical data of two unusual cases with chest CT or V-P scan imaging

\begin{tabular}{llllll}
\hline & Gender & Symptom & Details of assessment & Chest CT/VP scan & Tin \\
\hline Case 1 & $\mathrm{M}$ & Pleural pain & Pleural effusion on chest ultrasound & Normal VP scan & $7 \mathrm{~m}$ \\
Case 2 & $\mathrm{F}$ & Hemoptysis during acute COVID-19 & Negative D-dimers, abnormal 6MWT & Normal chest CTA & $6 \mathrm{~m}$ \\
\hline
\end{tabular}


CT - computed tomography, VP scan - ventilation-perfusion scan

\section{Discussion}

Our study describes relatively mild and limited respiratory consequences of COVID-19 in our group of children. There are several results from our research. First, we found a relatively low risk of structural and/or functional abnormalities of the respiratory tract and clinical remission usually occurred within several months.

Second, we recognized four subgroups of respiratory post-COVID syndrome with different symptomatology in the studied cohort. Besides the standard post-COVID group, "sporty" subgroup was represented by children used to high-intensity physical activities. These subjects sensitively identified worsening in their physical performance, and their results were usually normal. This group is very probably under-reported due to observer bias. Children with no regular sports activity could miss discrete symptoms exertional dyspnea without regular physical activity. The third subgroup denotes probable incipient bronchial asthma. COVID-19 is not a potential trigger but may coincide with this frequent chronic diagnosis. Furthermore, the focused attention of parents on respiratory symptoms in children after COVID-19 may accelerate early examination by a pulmonologist. There was usually positive family history, atopic history, positive allergy panel, and an excellent response to anti-asthma treatment. This group could be over-reported due to detection bias by pulmonologists. The fourth subgroup includes „polymorphous" post-COVID with a wide variety of symptoms (headache, concentration deficiency, anxiety, hair loss, chest pain, cough, dyspnea, anxiety, tachycardia) which were noticed apart from the monitored respiratory symptoms. Adolescent girls highly predominate. Previous mental health problem in them or their close relatives was found in $60 \%$. This group reflects the complex consequences of COVID-19 pandemic in a very fragile age. In our study this group is very probably under-reported due to selection bias. Variety of their non respiratory problems may lead to referral to another specialist and not to a pulmonologist.

Our results are in accordance with other published work designed as case series. These studies confirmed existence of pediatric post-COVID syndrome and reported diverse symptoms and infrequent occurrence. The first paper on post-COVID-19 syndrome was published by Ludgvisson et al. in November 2020. They collected data from parental reports of five Swedish children with persistent symptoms lasting two months or more after acute COVID infection. Parents contacted authors on an Internet-based social media forum. These children's symptoms lasted for 6-8 months after their clinical diagnoses of COVID-19 and included fatigue, dyspnea, cardiac palpitations, chest pain, headache, muscle weakness, dizziness, and sore throat. Subsequently, Buonsenso et al. published a cross-sectional study in which 129 children aged 6-16 years diagnosed with COVID-19 between March and November 2020 were interviewed by phone or in an outpatient clinic.

More than one-third reported one or two lingering symptoms four months or more after COVID-19 infection, and a further one-quarter had three or more symptoms. Insomnia, fatigue, muscle pain, and persistent cold-like complaints were common. In a prospective cohort study of Monteni et al., about $1.8 \%$ of 1379 children experienced symptoms for at least 56 days after acute COVID, according to data reported by adult proxy via mobile application Radtke et al. compared in a longitudinal cohort study the occurrence of at least one symptom lasting beyond 12 weeks in seropositive and seronegative children (4\% vs $2 \%$ ). Parents reported symptoms via an online questionnaire. The most common complaints included fatigue, difficulty concentrating, and increased sleepiness. Brackel et al. conducted a Dutch national survey on 89 children with long COVID identified by pediatric departments. The most frequent symptoms comprised fatigue, dyspnea, and concentration difficulties; authors described in detail a case series of six children.

To our knowledge, our work is a first prospectively planned study focused on respiratory aspects of pediatric post-COVID. Our data suggest lower risk of functional lung impairment compared to published data in adults.

The strength of our study includes the cooperation of centers collecting data from all regions of the Czech Republic and timing of the survey following a peak of the critical epidemic situation in the Czech Republic 
with highest COVID-19 related mortality worldwide. Actual evidence of 1.67 million confirmed SARS infections as of August 2021 may assure adequately large sample for monitoring pediatric post-COVID.

The main limitations comprise differences in laboratory equipment and software between centers, and a rather short follow-up to allow for timely publishing of the results.

\section{Conclusion}

According to this first data, the long-term respiratory impact of COVID-19 appears relatively mild with a favorable prognosis in children. Further studies with more subjects and longer follow up are needed. The actual test panel seems effective for the evaluation of structural and functional abnormities of the respiratory tract in pediatric post-COVID.

Ethical approval Approved by Ethics Committee with the multicentre competence of the Institute for Clinical and Experimental Medicine and the Thomayer University Hospital (A-21-01).

Presented at The design of this study was presented at the 19th Conference of Paediatric Pulmonology in Prague in April 2021 as an oral presentation.

Contributors DK: planning and execution of the study, statistical analysis and drafting the manuscript. TJ: planning and execution of the study, revising the manuscript. DK and TJ contributed equally. PP: planning the study and revising the manuscript. All the authors agreed with the submission of this manuscript.

Acknowledgement: We want to cordially thank our colleagues who participated in our study with patient recruitment and data sharing: Látalová Vendula, Department of Paediatrics, Faculty of Medicine, Palacký University and University Hospital Olomouc, Ř́íhová Renata, Department of Paediatrics, University Hospital Bulovka, Prague, Mohylová Veronika, Department of Paediatrics, Faculty of Medicine, University of Ostrava and University Hospital Ostrava, Martinů Vendula, Department of Paediatrics, 2nd Faculty of Medicine, Charles University in Prague and University Hospital Motol, Prague, Kreslová Marcela, Department of Pediatrics, University Hospital and Faculty of Medicine in Pilsen, , Červíček Stanislav, Department of Pediatrics, Hospital České Budějovice, Cipra Adam, Department of Pediatrics, Krajská zdravotní, a.s. Ústí nad Labem.

Funding This work was supported by the Charles University in Prague [UNCE 204064].

\section{References:}

Table 1. Clinical symptoms, their duration, and abnormal results in all subjects and post-COVID subgroups

Table 2. Persistence of reported symptoms in months within post-COVID until remission

Table 3. Details and clinical data of two unusual cases with chest CT or V-P scan imaging 